\title{
SynDiQuAss - Synchronisierung von Digitalisierung, Qualitätssicherung und Assistenzsystemen
}

\author{
Tobias Rusch, Michael Hueber, Florian Kerber, Robin Sochor, Klaus Fink, \\ Hermann Klug, Benedikt Stelzle und Massimo Romanelli
}

\subsection{Die Motivation der beteiligten Partner}

Das Konsortium des Forschungsprojektes SynDiQuAss besteht aus fünf Partnern. Die Hochschule Augsburg mit dem Technologietransferzentrum Nördlingen (TTZ) und das Fraunhofer Institut für Gießerei-, Composite- und Verarbeitungstechnik IGCV mit Sitz in Augsburg sind dabei als wissenschaftliche Partner im Projekt aktiv. Hauptzielsetzungen des TTZ sind angewandte Forschungsprojekte und Technologietransferkooperationen zur Unterstützung vorwiegend regional ansässiger kleiner und mittelständischer Unternehmen des produzierenden Gewerbes bei der Umsetzung der digitalen Transformation der industriellen Produktion. Innerhalb des Fraunhofer IGCV beschäftigt sich eine Forschungsgruppe mit der Integration und Applizierung von Assistenzsystemen an produktionstechnischen Arbeitsplätzen in unterschiedlichen Unternehmensbereichen wie

T. Rusch $(\bowtie) \cdot$ M. Hueber · F. Kerber

Hochschule Augsburg, Technologietransferzentrum Nördlingen,

Nördlingen, Deutschland

R. Sochor $\cdot$ K. Fink

Fraunhofer-Institut für Gießerei-, Composite- und Verarbeitungstechnik IGCV.,

Augsburg, Deutschland

H. Klug

SPN Schwaben Präzision Fritz Hopf GmbH, Nördlingen, Deutschland

B. Stelzle

Ohnhäuser GmbH, Wallerstein, Deutschland

M. Romanelli

paragon semvox GmbH, Kirkel-Limbach, Deutschland

(C) Der/die Autor(en) 2021

W. Bauer et al. (Hrsg.), Arbeit in der digitalisierten Welt,

https://doi.org/10.1007/978-3-662-62215-5_19 
der Logistik und Montage. Bei beiden Partnern lagen zu Projektbeginn bereits umfangreiche Kompetenzen im Bereich von Assistenzsystemen in der Produktion vor. Diese Erfahrungswerte konnten im Laufe des Projekts SynDiQuAss u. a. in den Bereich der Qualitätssicherung übertragen werden.

Die beiden mittelständischen Industriepartner SPN Schwaben Präzision Fritz Hopf $\mathrm{GmbH}$ in Nördlingen und Ohnhäuser $\mathrm{GmbH}$ in Wallerstein liefern im Projekt zwei unterschiedliche Anwendungsszenarien, anhand derer die Herausforderungen bei der Digitalisierung von variantenreichen Produktfamilien, kleinen Losgrößen und manuellen Montageprozessen exemplarisch untersucht werden können. Die SPN verfolgt im Rahmen von SynDiQuAss Ziele in den Bereichen Personalmanagement, Qualitätssicherung und Arbeitsplatzgestaltung. Durch den Einsatz von digitalen Assistenzsystemen sollen neue MitarbeiterInnen schneller für die selbstständige Montage komplexer mechatronischer Produktgruppen befähigt werden. Im Bereich der Qualitätssicherung sollen Fehlerquoten in der Montage, die aufgrund der hohen Variantenvielfalt und konstruktiven Besonderheiten wie besonders niedriger Toleranzen auftreten können, reduziert werden. Außerdem verspricht sich die SPN in der Arbeitsplatzgestaltung eine Reduktion des Flächenbedarfs durch Flexibilisierung der Fertigungslinien und deren Umgestaltung. Zusammenfassend lässt sich für die SPN sagen, dass weiterhin die Absicherung von standardisierten Montageprozessen durch die WerkerInnen auf Basis von detaillierten Prozessbeschreibungen (Wissensmanagement) und die Dokumentation von Prozessen zur Rückverfolgbarkeit im Qualitätsmanagement essenziell ist. Nicht zuletzt beabsichtigt die SPN durch die Assistenzsysteme eine spürbare Effizienzsteigerung von Montageprozessen durch Fehlerreduktion, beschleunigte Einarbeitung und Prozessabwicklung sowie die Entlastung von WerkerInnen von kognitiven Anforderungen bei der korrekten Einhaltung von Vorgaben durch den Montageprozess. Der Forschungsanreiz der Firma Ohnhäuser liegt darin, bereits existierende Montagearbeitsplätze nach neuesten Standards der Industrie 4.0 umzugestalten. Als Hauptziel hat sich das mittelständische Unternehmen die digitale Vernetzung der Anlagen und eine Automatisierung der Prozesse gesetzt. Dadurch soll die Prozesssicherheit unabhängig von Personalfluktuationen und Produktbzw. Verarbeitungsvarianten gesteigert werden. Für die Einhaltung vorgegebener Qualitätsstandards ist der Einsatz eines kollaborierenden Robotersystems vorgesehen. Die Realisierung wäre ohne Beteiligung an diesem Forschungsprojekt für Ohnhäuser nicht darstellbar. Als typische Repräsentanten mittelständischer Unternehmen im ländlichen Raum sind die im Projekt betrachteten Anwendungsszenarien branchenübergreifend übertragbar, sodass die Projektergebnisse auch nach Laufzeitende verwertbar sind.

Als Technologiepartner bringt die paragon semvox $\mathrm{GmbH}$ Kenntnisse aus dem Bereich der multimodalen Assistenzsysteme zur Prozessunterstützung in Produktion und Logistik ein. Das Technologieunternehmen entwickelt die Softwareplattform zur Integration physischer und kognitiver Assistenzsysteme mit multimodalen Interaktionsmöglichkeiten. Dabei ist die optimale Einbindung in die bestehenden betrieblichen Arbeitsabläufe der Industriepartner ein wichtiges Entwicklungsziel. Das Gesamtsystem 
muss flexibel und schnell an kurzfristige Veränderungen im Produktionsablauf angepasst werden können und universelle Schnittstellen sowohl für verschiedene Assistenzsystemkomponenten als auch zu relevanten Unternehmensbereichen wie der Produktionsplanung implementieren.

\subsection{Zielsetzungen und Vorgehen im Projektverlauf}

In der manuellen Montage steht der Mensch im Mittelpunkt. Die auszuführenden manuellen Tätigkeiten des Menschen können durch kognitive- und physische Assistenzsysteme unterstützt werden, sodass die Fähigkeiten des Menschen vorteilhaft mit den besonderen Eigenschaften von Maschinen kombiniert werden [1]. Die Entwicklung und Evaluierung derartiger digitaler Assistenzsysteme ist ein wesentlicher Arbeitsschwerpunkt im Projekt SynDiQuAss. Die Assistenzsysteme werden dazu in die bestehenden Arbeitsplätze im Bereich der bisher nicht automatisierten Montage integriert. Der Fokus liegt auf der systematischen Auswahl geeigneter Komponenten zur kognitiven und physischen Assistenz und deren Applizierung an spezifizierten Arbeitsplätzen für die Montage variantenreicher Produktfamilien, die exemplarisch aus den Anwendungsfällen der beteiligten mittelständischen Industriepartner ausgewählt wurden. Bei diesem Vorgehen wird großer Wert auf die Einbindung der MitarbeiterInnen bei der Optimierung der einzelnen Funktionalitäten gelegt, um so Akzeptanz für die Nutzung dieser Systeme zu fördern. Das in Abschn. 19.4 detaillierter beschriebene partizipative Vorgehensmodell spielt im Projektverlauf für die Integration der Assistenzsysteme eine tragende Rolle, um insbesondere die Schnittstellen der Mensch-Maschine-Interaktion [2] bedarfsgerecht auszulegen.

Die Betrachtung der Wirtschaftlichkeit und Produktivitätsauswirkungen bei den Industriepartnern wurde an die Evaluierungsphasen angehängt. Ein wesentlicher Aspekt dabei ist die Anpassbarkeit an große Variantenvielfalt in den einzelnen Produktfamilien bei kleinsten Losgrößen, um die spätere Verwertbarkeit gerade in KMUs zu sichern. Um diesen Anforderungen gerecht zu werden, wurden im Projekt formale Methoden zur Modellierung des gesamten Montageprozesses für die montagetechnische Planung und speziell für die Gestaltung eines Montagearbeitsplatzes mit integrierten Assistenzsystemen angewendet. Die in [3] beschriebene Methode nutzt UML-Diagramme zur strukturierten Beschreibung des Variantenraums von Produktfamilien und der daraus resultierenden Varianz der Montageschritte. Die softwaretechnische Umsetzung des Modellierungsansatzes erfolgt über die im Projekt entwickelte Software Assistent/Editor, in der Montageschritte, Produktkomponenten und Assistenzsystemfunktionalitäten verknüpft und über Schnittstellen zu anderen Unternehmensbereichen bzw. Assistenzsystemkomponenten mit relevanten Daten wie CAD-Informationen etc. versorgt werden. So kann kognitive und physische Unterstützung bei der Montage von individuellen Varianten mit geringen Stückzahlen mit einem möglichst geringen Integrationsaufwand der Systeme für die anwendenden Unternehmen bereitgestellt werden. Um 
im Anwendungsbeispiel eines Industriepartners möglichst viele Gesichtspunkte der manuellen Getriebemontage darzustellen, wurde im Modell der gesamte Montageprozess in seine elementaren Prozessschritte unterteilt. Die subjektive Bewertung der möglichen Einsatzpotenziale für digitale Assistenzsysteme erfolgte gemeinschaftlich mit den Industriepartnern in Workshops [4].

Das strukturierte und planvolle Vorgehen im Forschungsprojekt SynDiQuAss unter der Zielsetzung der Transformation von digitalisierten Assistenzsystemen in den realen Arbeitsprozess der manuellen Montage von Getrieben soll dazu beitragen, die Produktivität der neuen Prozesse zu steigern. Für die Bewertung der Produktivität und Wirtschaftlichkeit der entwickelten Lösungsansätze liefern die in Tab. 19.1 zusammengefassten Kriterien und Bewertungsmethoden quantifizierbare Größen.

Berücksichtigt werden zum einen menschliche Stärken wie Intuition, Erfahrung, Flexibilität, subjektives Entscheiden und Urteilen und zum anderen die erkannten Vorteile der eingesetzten Assistenzsysteme. Dazu zählen Wiederholgenauigkeit und Präzision, aber auch Präzision bei hohen Geschwindigkeiten und Funktionalität im Dauerbetrieb [5]. Assistenzsysteme wurden deshalb nicht mit der Zielsetzung appliziert, den gesamten Prozess zu assistieren oder gar zu automatisieren, sondern lediglich eine Teilmenge der Arbeitsschritte, in denen der Einsatz nach den Kriterien sinnvoll erscheint. Wie bei [6] und [7] beschrieben, tragen physische Assistenzsysteme zu einer reduzierten körperlichen Belastung bei und stellen die motorische Ausführbarkeit der Arbeitsaufgabe sicher. Kognitive Assistenzsysteme unterstützen den Werkenden bei der Ausführung der Montageaufgabe durch die situative Bereitstellung aller notwendigen Informationen. Die Bewertungsgrundlage dafür lieferten die Akzeptanzuntersuchungen mit den betroffenen MitarbeiterInnen.

Für den mechanischen Aufbau des standardisierten Systemarbeitsplatzes wurde ein modularer Aufbau gewählt, wie er in [8] beschrieben ist. Jeder Bereich des Arbeitsplatzes wird für einzelne Aktivitäten im Montageprozess genutzt und der Arbeitsplatz so in spezielle Bereiche (Module) aufgeteilt. Dadurch können für die Anwendungsfälle einzelne Module für Assistenzsysteme ausgewählt und integriert werden. In Hinblick auf die zu erwartenden Belastungen der MitarbeiterInnen wurden zudem die arbeitswissenschaftlichen Grundlagen für die ergonomische Gestaltung nach DIN EN ISO

Tab. 19.1 Methoden zur Produktivitätsmessung

\begin{tabular}{l|l}
\hline Kriterium & Bewertungsmethode \\
\hline Arbeitszeit & MTM-Methode \\
\hline Risikobewertung & FMEA \\
\hline Transparenz und Flexibilität & Mitarbeiterbefragung in der Montage und Arbeitsvorbereitung \\
\hline Platz & Abmessung Arbeitsplatz - Vorher/Nachher-Vergleich \\
\hline Ergonomie und Akzeptanz & UTAUT-Methode \\
\hline
\end{tabular}




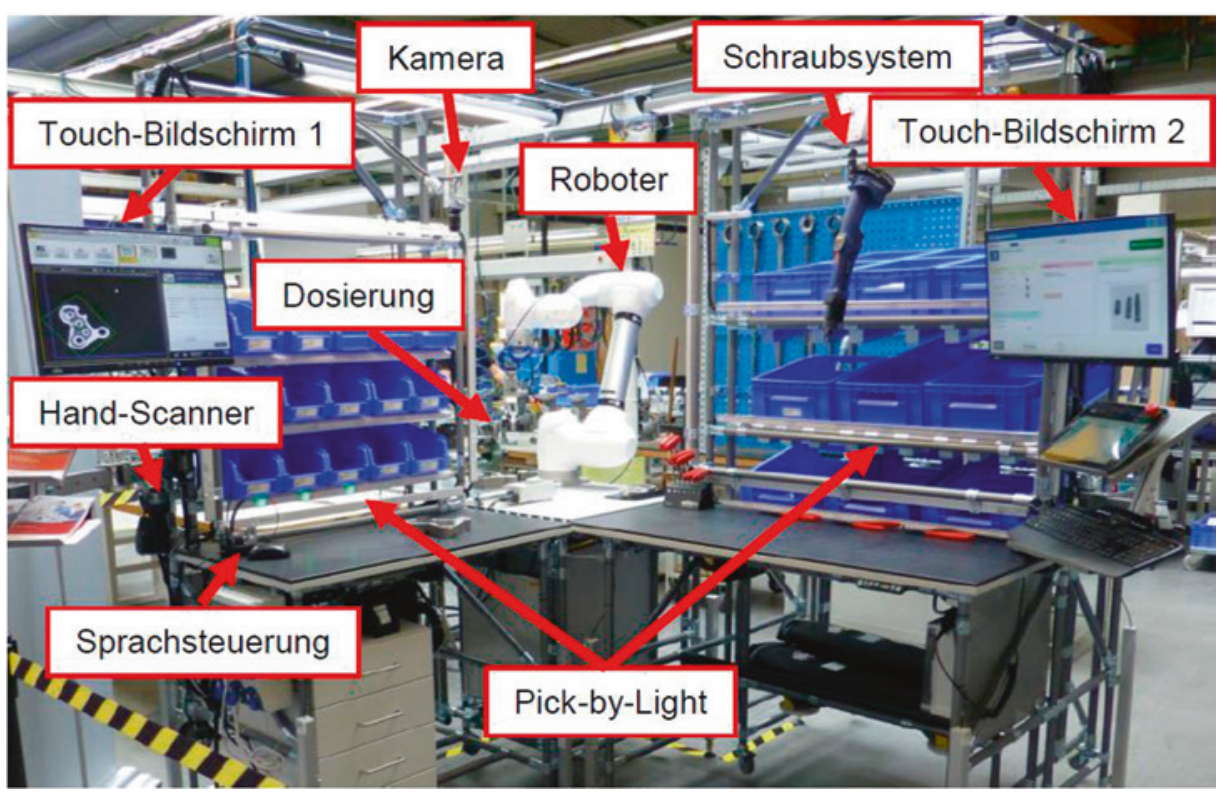

Abb. 19.1 Prototypischer Aufbau des Demonstratorarbeitsplatzes

6385 (2016) [9] berücksichtigt. Abb. 19.1 zeigt den prototypischen Aufbau des Montagearbeitsplatzes für den Anwendungsfall einer Getriebemontage.

Auch die Sicherheit an den Demonstratorarbeitsplätzen muss bei allen Testsystemen und Erprobungen jederzeit gewährleistet werden. Die im Projekt eingesetzten Technologien und Methoden dienen deshalb dazu, die Gefahren in der Arbeitsumgebung der WerkerInnen zu reduzieren. Dies gilt im Besonderen für den Einsatz der kollaborierenden Robotersysteme. Dazu mussten bestimmte roboterspezifische Gefährdungen mithilfe einer Risikobeurteilung berücksichtigt werden, beispielsweise Robotereigenschaften (Geschwindigkeit, Kraft oder Impuls) und die Position des Bedienpersonals zum Roboter. Gefahren bedingen dabei auch Werkstücke und Aufbauten im möglichen Wirkfeld des Roboters. Hinzu kommen prozessspezifische Gefährdungen wie hohe/niedrige Temperaturen oder Einschränkungen aufgrund der geforderten Verwendung von persönlicher Schutzausrüstung [1, 2].

Um bei der Umsetzung die großen Potentiale für Unternehmen von Anfang an zu nutzen, bedarf es eines Vorgehensmodells zur Prozessevaluierung. Mit diesem lässt sich die Integration der kognitiven und physischen Assistenzsysteme für die Montage auf Basis der Evaluierungen optimal strukturieren [4]. Durch die Anwendung und Verknüpfung verschiedener wissenschaftlicher Methoden entsteht das in Abb. 19.2 dargestellte Vorgehensmodell. Daraus resultiert eine schematische Handlungsempfehlung für mittelständische Unternehmen um digitale Assistenzsysteme in ihr Produktionsumfeld zu integrieren. 


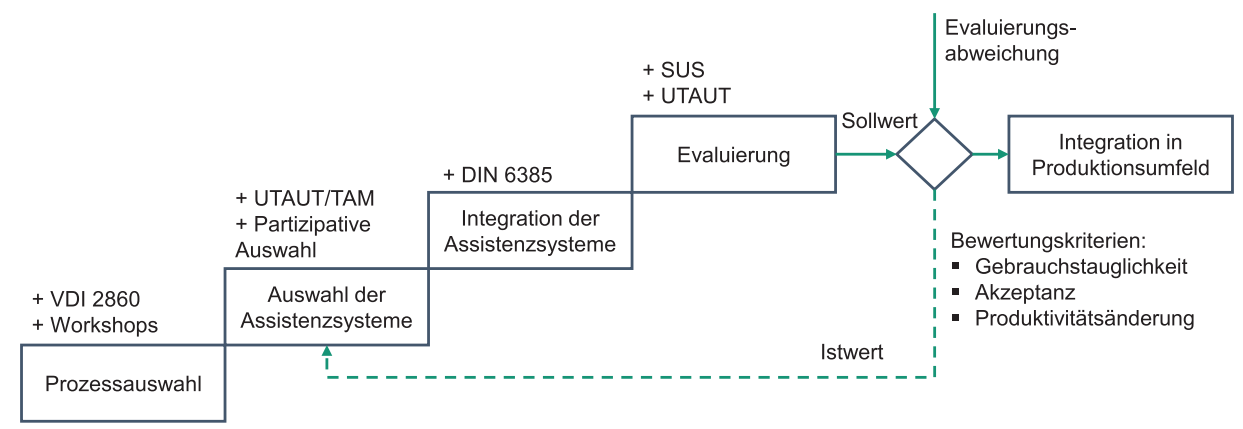

Abb. 19.2 Vorgehen zur Integration von Assistenzsystemen [4]

\subsection{Vorgehen zur partizipativen Entwicklung von Assistenzsystemen}

Die Erhebung des Status quo sowie die Entwicklung der Assistenzsysteme beziehen neben technischen Anforderungen auch die Betrachtung des Menschen mit dessen Anforderungen ein. Dies spielt insbesondere bei der Entwicklung von soziotechnischen Systemen eine wichtige Rolle [1, 10, 11]. Die Anpassung des Systems an die Arbeitsaufgaben sowie die Akzeptanz der Belegschaft gegenüber dem Assistenzsystem können dafür ausschlaggebend sein, ob die Implementierung Erfolg hat oder aufgrund von Ablehnung scheitert $[12,13]$. Denn es ist nicht alles, was denkbar und technisch realisierbar ist, auch im Sinne einer humanen Arbeitswelt vertretbar. Die Projektpartner verpflichteten sich daher vor Beginn der konkreten Tätigkeit in den Unternehmen auf ein Commitment. Dieses wurde gemeinsam erarbeitet und beinhaltet die Regeln für das Vorgehen im Projekt und auch die Gestaltung der Arbeitsplätze. Zusammen mit den Industriepartnern und deren MitarbeiterInnen wurden die Anforderungen an die zu entwickelnden Systeme erarbeitet.

Ein solches, partizipatives Vorgehen eignet sich, um spezifizierte Assistenzsysteme zu entwickeln. Dabei hat die aktive Einbindung der MitarbeiterInnen zwei Vorteile: Zum einen verfügen diese über Erfahrungswissen, das für die Entwicklung des Systems unter Berücksichtigung der Passung zur Arbeitsaufgabe notwendig ist. Zum anderen fördern frühzeitiges Informieren und aktives Einbinden der MitarbeiterInnen deren Bereitschaft zur Verantwortungsübernahme sowie deren Akzeptanz [14-16]. Im Projekt werden als Grundlage für ein partizipatives Entwicklungsvorhaben Workshops mit MitarbeiterInnen und Bereichsverantwortlichen durchgeführt sowie ein Montagearbeitsplatz bei den Industriepartnern vor Ort aufgebaut, der eine Auswahl an kognitiven Assistenzsystemen enthält, Abb. 19.3.

Im Vorfeld der Montageplatzkonzipierung wurden mehrere Marktanalysen durchgeführt, um die für die Montage relevanten Technologien zu identifizieren. Basierend auf den Marktanalysen wurde ein Querschnitt der relevanten Technologien ausgewählt 


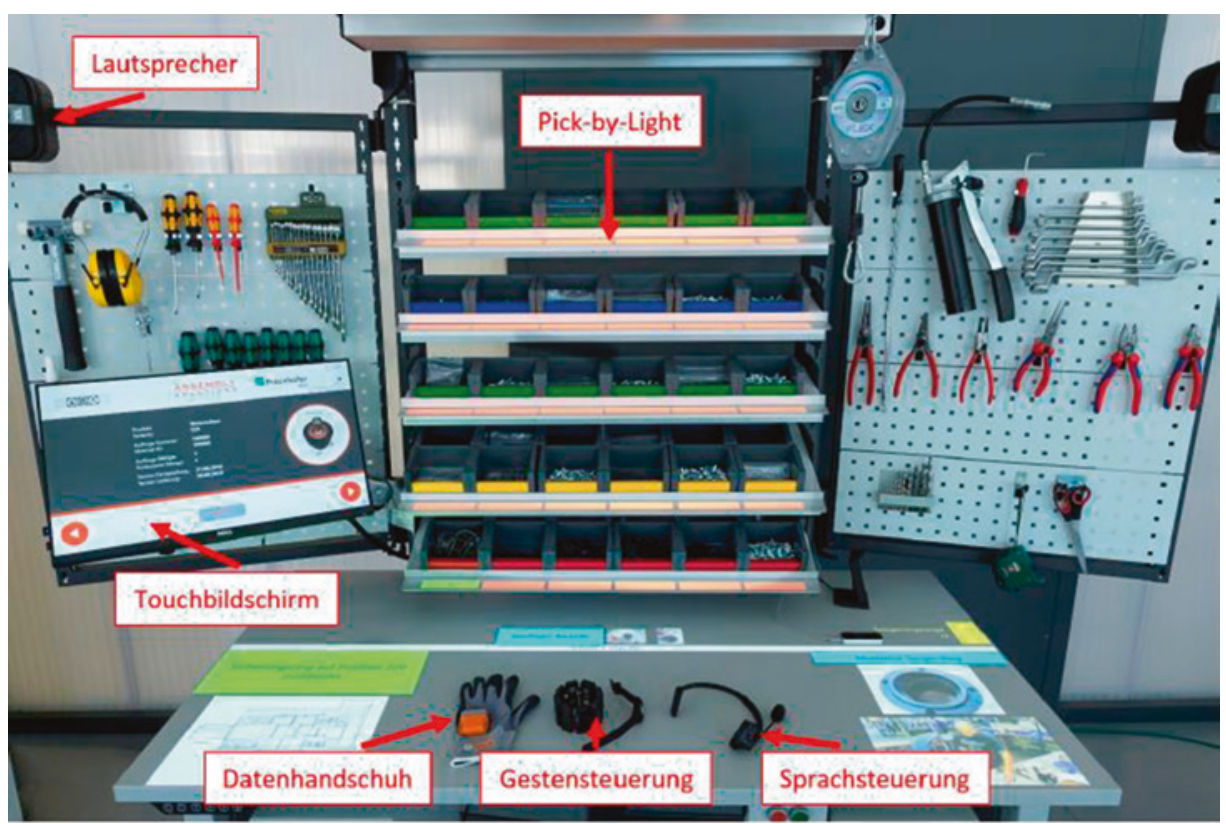

Abb. 19.3 Mobiler Montagearbeitsplatz mit Assistenzsystemen für partizipative Entwicklungsvorhaben [6]

und mit entsprechenden Komponenten (z. B. Gestensteuerung durch Leap Motion) am Montagetisch implementiert. Um die Komponenten möglichst realitätsgetreu testen zu können, wurden diese im Rahmen der beispielhaften Montage eines variantenreichen Kleingetriebes benutzt.

Der Demonstratorarbeitsplatz ist mobil und kann vor Ort im Unternehmen (z. B. direkt in der Montage) aufgebaut werden. In einer ca. 30-minütigen praktischen Einführung durch die WissenschaftlerInnen testeten die Probanden die Komponenten hinsichtlich ihrer Bedienbarkeit. Während dem Test wurden die Probanden außerdem dazu aufgefordert, ihre Gedanken zur Nutzung der Assistenzsysteme zu äußern. In einem abschließenden standardisierten Fragebogen wurden die unterschiedlichen Systeme hinsichtlich ihrer Einfachheit der Nutzung, Zuverlässigkeit und Nutzungsintention verglichen. Für eine bessere Verständlichkeit wurde der Fragebogen mit Bildern angereichert, welche die unterschiedlichen Komponenten direkt am Montagetisch zeigen. Mithilfe der gewonnen qualitativen und quantitativen Daten konnte im Anschluss ein für den Use Case spezifisches Assistenzsystem entwickelt werden, das von den MitarbeiternInnen befürwortete Komponenten enthält und zu den Bedingungen der Arbeitsaufgabe und -umgebung passt. Zudem erhielten die MitarbeiterInnen die Möglichkeit, sich über aktuelle Technologien zu informieren und sich tiefer mit der Bedienung unterschiedlicher Komponenten auseinanderzusetzen. Schließlich wurde 
eine Diskussionsgrundlage für das weitere Entwicklungsvorgehen bei allen beteiligten Personengruppen geschaffen. Bei der Firma Ohnhäuser wurde der DemonstratorArbeitsplatz über einen Zeitraum von drei Tagen in der Montage aufgebaut. Die Testergebnisse dienten als Grundlage für die in den folgenden Kapiteln ausgeführten Konzeptionierung hybrider Assistenzsysteme.

Die Gebrauchstauglichkeit des entwickelten Montagesystems lässt sich mit der etablierten und empirischen Methode, System Usability Scale (SUS), quantitativ analysieren. Für die Methode ist zuvor eine Auswahl der Nutzergruppe, der zu erledigenden Aufgaben, sowie die Charakteristiken des Umfelds notwendig. Die Befragung umfasst zehn standardisierte Fragen [17]. Für die Untersuchung der Akzeptanz und des Nutzens des Montagesystems findet die Methode Unified Theory of Acceptance and Use of Technology (UTAUT) Anwendung. Diese betrachtet die möglichen Effekte der Leistungserwartung, Aufwandserwartung und sozialen Einflüsse. Diese werden den indirekten Variablen wie beispielsweise Geschlecht, Alter und Erfahrung gegenübergestellt. Beide Methoden wurden im Zuge der Evaluierung anhand eines Fragebogens kombiniert [4].

Nach der Evaluierung des Montagesystems hinsichtlich der Gebrauchstauglichkeit, Akzeptanz und des Nutzens erfolgte der Abgleich der Ergebnisse anhand des Soll-/ Istwert-Vergleichs. Werden die Sollwerte erreicht, kann das Montagesystem in das Produktionsumfeld ausgerollt werden. Die zu erreichenden Sollwerte wurden gemeinschaftlich innerhalb des Projektteams und der Stakeholder definiert. Das Vorgehensmodell läuft iterativ ab, bis die Erreichung der Sollwerte durch eine Evaluierung bestätigt wird [4].

\subsection{Forschungsergebnisse des umgesetzten Anwendungsfalls}

Für die Evaluierung des unter Abschn. 19.2 beschriebenen Vorgehensmodell wurde ein spezifischer Fragebogen entwickelt. Dieser beinhaltet in Summe 23 Fragen unter Berücksichtigung der vorgestellten Methoden. Die Evaluierung des entwickelten Montagesystems fand bei der Fa. SPN, ein Forschungspartner und mittelständisches Maschinenbauunternehmen, statt. Wie in dem Vorgehen in Abschn. 1.3 beschrieben, wurden in Summe 29 Probanden für die Evaluierung ausgewählt. Dabei richtete sich die Auswahl der Probanden nach der zukünftigen Benutzergruppen der Studenten und Azubis $(n=12)$, Monteure mit einer geringeren Berufserfahrung von einem Jahr $(n=8)$ und Monteure mit mehr als einem Jahr Berufserfahrung $(n=9)$ in der manuellen Montage. Die Aufgabe der Probanden war der vollständige Zusammenbau von sieben Getriebebauteilen gleicher Ausführung in Reihenfolge unter der Verwendung von kognitiven und physischen Assistenzsystemen. Im Anschluss an die Montage der sieben Getriebe wurden die ProbandInnen anhand des entwickelten Fragebogens befragt. Dabei wurde für die SUS- und UTAUT-Methode eine Likert-Skala ( $1=$ stimme zu; $2=$ stimme 
Tab. 19.2 Evaluierungsergebnisse nach der Methodik System Usability Scale

\begin{tabular}{l|l|l|l}
\hline Fragen & $\mathrm{n}$ & Mittelwert & Standardabweichung \\
\hline $\begin{array}{l}\text { Ich kann mir sehr gut vorstellen, das System } \\
\text { regelmäßig zu nutzen }\end{array}$ & 29 & 1,51 & 0,72 \\
\hline Ich empfinde das System als unnötig komplex & 29 & 4,28 & 0,74 \\
\hline Ich empfinde das System als einfach zu nutzen & 29 & 1,59 & 0,49 \\
\hline $\begin{array}{l}\text { Ich denke, dass ich techn. Support brauchen würde, um } \\
\text { das System zu nutzen }\end{array}$ & 29 & 3,63 & 1,19 \\
\hline $\begin{array}{l}\text { Ich finde, dass die ver. Funktionen des Systems gut } \\
\text { integriert sind }\end{array}$ & 29 & 1,72 & 0,58 \\
\hline $\begin{array}{l}\text { Ich finde, dass es im System zu viele Inkonsistenzen } \\
\text { gibt }\end{array}$ & 29 & 4,28 & 0,64 \\
\hline $\begin{array}{l}\text { Ich kann mir vorstellen, dass die meisten Leute das } \\
\text { System schnell zu beherrschen lernen }\end{array}$ & 29 & 1,28 & 0,45 \\
\hline \begin{tabular}{l} 
Ich empfinde die Bedienung als sehr umständlich \\
\hline $\begin{array}{l}\text { Ich habe mich bei der Nutzung des Systems sehr sicher } \\
\text { gefühlt }\end{array}$
\end{tabular} & 29 & 4,21 & 0,85 \\
\hline $\begin{array}{l}\text { Ich musste eine Menge Dinge lernen, bevor ich mit } \\
\text { dem System arbeiten konnte }\end{array}$ & 29 & 3,97 & 0,72 \\
\hline
\end{tabular}

eher $\mathrm{zu} ; 3=$ weder noch; $4=$ stimme eher nicht $\mathrm{zu} ; 5=$ stimme nicht $\mathrm{zu}$ ) verwendet (Tab. 19.2).

Zusammenfassend lässt sich anhand der SUS-Methodik festhalten, dass sich die ProbandInnen $(n=29)$ die regelmäßige Nutzung des Montagesystems vorstellen können. Die Auswahl der kognitiven und physischen-Assistenzsysteme wird als passend bewertet und deren Integration als gut befunden. Dies führt zu einer schnellen Beherrschung des Systems während der Anwendung (Tab. 19.3).

Anhand der UTAUT-Methodik lässt sich darauf schließen, dass die Probanden $(n=29)$ nach einer subjektiven Beurteilung die Kontrolle über das Montagesystem haben und alle notwendigen Informationen vom System bereitgestellt bekommen. Zudem sind die Probanden davon überzeugt, dass die Qualität des Bauteils/der Baugruppe durch den Montageassistenten erhöht werden kann.

\subsection{Weitergehende Forschungsansätze aus den Ergebnissen von SynDiQuAss}

In den folgenden Monaten strebt das Konsortium an, die Ergebnisse der ersten Evaluationsphase des prototypischen Montagearbeitsplatzes mit integrierten Assistenzsystemen vollständig auszuwerten und daraus Optimierungen des bestehenden Systems insbesondere im Hinblick auf die Ergebnisverwertung, die Übertragbarkeit der ein- 
Tab. 19.3 Evaluierungsergebnisse nach der Unified Theory of Acceptance and Use of Techno$\log y$

\begin{tabular}{|c|c|c|c|}
\hline Fragen & $\mathrm{n}$ & Mittelwert & Standardabweichung \\
\hline $\begin{array}{l}\text { Meine Vorgesetzten sind davon überzeugt, dass ich das } \\
\text { System verwenden muss }\end{array}$ & 29 & 2,00 & 2,29 \\
\hline $\begin{array}{l}\text { Ich habe das Gefühl die Kontrolle über das System zu } \\
\text { haben }\end{array}$ & 29 & 1,62 & 0,72 \\
\hline $\begin{array}{l}\text { Ich erhalte von dem System alle notwendigen } \\
\text { Informationen }\end{array}$ & 29 & 1,48 & 0,81 \\
\hline Das System erleichtert mir die körperliche Arbeit & 29 & 2,17 & 1,12 \\
\hline $\begin{array}{l}\text { Das System macht das Arbeiten sicherer. (Arbeits- } \\
\text { sicherheit) }\end{array}$ & 29 & 1,79 & 0,80 \\
\hline Das System schränkt mich in meiner Arbeitsweise ein & 29 & 4,03 & 0,89 \\
\hline $\begin{array}{l}\text { Das System erhöht die Qualität des Bauteils/der Bau- } \\
\text { gruppe }\end{array}$ & 29 & 1,62 & 0,72 \\
\hline
\end{tabular}

gesetzten Methodiken sowie die Wirtschaftlichkeitsanalyse abzuleiten. Dabei sollen insbesondere noch Aspekte der Arbeitssicherheit des Demonstratorarbeitsplatzes berücksichtigt werden. Speziell das Fehlermanagement der integrierten Assistenzsysteme, die Arbeitssicherheit in der Interaktion mit kollaborierenden physischen Assistenzsystemen und die Vorgehensweise für WerkerInnen bei auftretenden Störungen des Systems sollen weiterentwickelt werden. Das Konsortium verspricht sich dadurch weitere praktisch verwertbare Handlungsleitfäden für spezifizierte Montagearbeitsplätze. Des Weiteren soll die wirtschaftliche Auswertung im Sinne des Produktivitätsmanagements unter Einbeziehung der Optimierungen vertieft werden. Dazu sollen eine aktualisierte Prozess-FMEA des spezifizierten Montagearbeitsplatzes und eine Wirtschaftlichkeitsbetrachtung anhand des untersuchten Anwendungsfalls exemplarisch durchgeführt werden. Die von paragon semvox mithilfe aller Projektpartner entwickelte Software zur Erstellung von digitalen Arbeitsabläufen (Editor) soll in der weiteren Projektlaufzeit von erfahrenen MitarbeiterInnen in den Industrieunternehmen separat evaluiert werden. Dadurch lässt sich die Software noch besser an die Anforderungen in realen Arbeitsbedingungen anpassen. Die zusätzlich gewonnenen Erkenntnisse sollen für das Rollout in den beteiligten Partnerunternehmen z. B. in die Unternehmensbereiche Logistik, Versand und Einzelteilfertigung genutzt werden und in einem Leitfaden zum partizipativen Vorgehensmodell der Integration von Assistenzsystemen an Montagearbeitsplätzen veröffentlicht werden.

Das Projekt SynDiQuAss hat sich auf den Unternehmensbereich der Montage fokussiert. Das entwickelte Vorgehensmodell und die eingesetzten Methoden und Technologien bietet jedoch Potenziale, in andere Unternehmensbereiche übertragen zu werden. Damit könnten zukünftig zum einen Produktivitätssteigerungen und Qualitätsverbesserungen entlang der gesamte Wertschöpfungskette in KMUs realisiert, zum 
anderen die für den Einsatz von Data Analytics und KI-basierter Verfahren notwendige innerbetriebliche Vernetzungsarchitektur und Datenplattform installiert werden. In diesem Kontext ergeben sich folgende offene Forschungsfragen:

- Inwiefern kann die Digitalisierung die Vernetzung zwischen Mitarbeitern im Unternehmen entlang des Wertschöpfungsprozesses unterstützen und dadurch Produktivität erhöhen?

- Wie kann im Mittelstand im Bereich des Wissenstransfers ein intelligenter Informationsfluss durch Assistenzsysteme und Anreizsysteme stattfinden?

- Wie muss ein flexibles Wissensmanagement mit Hilfe von Anreizsystemen gestaltet und mit digitalen Assistenzsystemen umgesetzt werden, damit Mitarbeiter anwendungsfallspezifisch Unterstützung erhalten?

- Welches Vorgehensmodell und welche Algorithmen eigenen sich für die automatisierte Erstellung von Prozessbeschreibungen basierend auf den vorhanden Entwicklungs- und Produktdaten?

- Wie können lernende Algorithmen an prozessintegrierten Prüfstationen wiederholgenaue und nachverfolgbare Aussagen über die aktuelle Produktqualität liefern und prädiktiv eingesetzt werden, um Fehlerfälle zu reduzieren?

- Wie kann der Materialfluss innerhalb der gesamten Produktion transparenter verfolgt und bedarfsgerechter gesteuert werden?

Diese Forschungsfragen stellen die Grundlage für Nachfolgeprojekte dar, die auf den Ergebnissen von SynDiQuAss aufbauen können.

\subsection{Publikationen und Veranstaltungen}

Im Laufe des Forschungszeitraums entstanden folgende Publikationen der Projektergebnisse:

- Fink, K., Rusch, T., Merkel, L., Sochor, R., Kerber, F., Reinhart, G.: Ein Vorgehensmodell zur Prozessevaluierung zur Integration ausgewählter kognitiver und physischer Assistenzsysteme am Montagearbeitsplatz 4.0 im Mittelstand, 66. Frühjahrskongress der Gesellschaft für Arbeitswissenschaft e. V., In: GfA-Press, 2020

- Riegel, A.: Assistance systems for process - integrated quality control at assembly workplaces Developing an inspection plan, In: Pro Business digital, 2019

- Sochor, R., Riegel, A., Merhar, L., Rusch, T., Kerber, F., Braunreuther, G., Reinhart, G.: Kognitive und physische Assistenz in der Montage, wt-online - Ausgabe 3-2019, S. 122-127, 2019

- Rusch T., Kerber F.: Prozessmodellierung zur Integration von Assistenzsystemen an Montagearbeitsplätzen. 65. Frühjahrskongress der Gesellschaft für Arbeitswissenschaft e. V., In: GfA-Press, 2019 
- Rusch T., Riegel A., Kerber F., Romanelli, M., Quitter T., Reinert M., Klug H.: Spezifikation und Umsetzungskonzept für standardisierte Montagearbeitsplätze mit integrierter Assistenzfunktion. Arbeit in der digitalisierten Welt. Stand der Forschung und Anwendung im BMBF-Förderschwerpunkt, In: Fraunhofer IAO, 2019

- Sochor R.; Riegel A.; Merhar L.; Rusch T.; Merkel L.; Kerber F.; Braunreuther S.; Reinhart G.: Kognitive und physische Assistenz in der Montage. Einsatzmöglichkeiten kombinierter Assistenz an Systemarbeitsplätzen. wt werkstatttechnik online, In: VDI Fachmedien GmbH, 2019

- Riegel A., Kerber F.: Assistierte Qualitätssicherung. Fachtagung Mechatronik, In: VDI Fachmedien GmbH, 2019

- Rusch, T.: Projekt SynDiQuAss - Assistenz für den Monteur von Morgen, In: Pro Business digital, 2019

- König, M., Stadlmaier, M., Rusch, T., Sochor, R., Merkel, L., Braunreuther, S., Schilp, J.: MA²RA - Manual Assembly Augmentes Reality Assistent, IEEM 2019

- Merhar L., Berger C., Braunreuther S., Reinhart G. (2019) Digitization of Manufacturing Companies: Employee Acceptance Towards Mobile and Wearable Devices. In: Ahram T. (eds) Advances in Human Factors in Wearable Technologies and Game Design. AHFE 2018. Advances in Intelligent Systems and Computing, vol 795. Springer, Cham

Die Projektziele und -ergebnisse wurden in verschiedenen Veranstaltungen sowohl für allgemeines Publikum als auch auf Fachtagungen präsentiert, um die Öffentlichkeitsarbeit und spätere Verwertung zu fördern.

- Sochor, R.: Current Knowledge Management in Manual Assembly - Further Development by the Analytical Hierarchy Process, Incentive and Cognitive Assistance Systems, CPSL Conference on Production Systems and Logistics 18.03.2020

- Sochor, R.: Digitaler Montagetisch, Schwerpunktgruppentreffen, Lemgo, 25.09.2019

- Kerber, F.: Assistenz in der Montage - Wie Cobots die Arbeitsplätze der Zukunft verändern, 8. Augsburger Technologietransfer-Kongress, 26.03.2019

- Kerber, F.: „Assistenzsysteme in der Produktion“ als Beitrag zur European Robotics Week, Augsburg, 18.11.2018

- Kerber, F.: Digitalization on the shopfloor - the SME perspective, Fachkonferenz im Technologiezentrum Augsburg anlässlich der Informationsreise "Industrie 4.0" einer slowenischen Delegation, 08.10.2018

- Hueber, M., Kerber, F: „Einsatz von Assistenzsystemen in der Montagetechnik“, Nördlingen, 01.02.2018

- Rusch, T.: Projekt SynDiQuAss - Assistenz für den Monteur von Morgen, Applied Research Conference 2018, Deggendorf, 10.07.2018

- Merkel, L.: Komplexität und Assistenzfunktionen in der manuellen Montage, MONTEXAS 4.0 Workshop, Lemgo, 15.02.2018 
Während der Projektlaufzeit nahm das Konsortium an verschiedenen Veranstaltungen teil und präsentierte die Forschungsergebnisse. Das Fraunhofer IGCV war auf den Hannover Messen 2018 und 2019 vertreten, um dort u. a. den selbst entwickelten Assistenzsystemdemonstrator vorzustellen. Auf der Arbeitsforschungstagung „Arbeitswelten der Zukunft" 2018 in Stuttgart war das Projekt mit einem eigenen Stand vertreten. Anlässlich der Feiern zum 100jährigen Firmenjubiläum des Projektpartners SPN Schwaben Präzision im September 2019 wurde der spezifizierte Demonstratorarbeitsplatz Fachbesuchern und der Öffentlichkeit präsentiert. Zudem wurde der Demonstrator zum „Tag des offenen Labors“" am Hochschulzentrum Donau-Ries ausgestellt.

\section{Projektpartner und Aufgaben}

- Hochschule Augsburg - Technologietransferzentrum, Nördlingen

Errichtung eines Prototypen für Arbeitsplätze mit geringem Automatisierungsgrad und Analyse von Möglichkeiten zur Unterstützung der WerkerInnen durch Mensch-Maschinen- Kollaborationen

- Fraunhofer-Einrichtung für Gießerei-, Composite- und Verarbeitungstechnik IGCV, Augsburg

Nachhaltige und signifikante Produktivitäts- und Flexibilisierungssteigerung in deutschen Fertigungsbetrieben sowie vertikale und horizontale Integrationsfähigkeit von Arbeitsprozessen durch gesteigerte Digitalisierung

- paragon semvox GmbH, Limbach/Kirkel

Weiterentwicklung und Etablierung digitalisierter Assistenzsysteme in der Produktion. Generierung neuer Forschungsideen und -potenziale

- Ohnhäuser GmbH, Wallerstein

Neue Anregungen und Erkenntnisse zu Innovationsmöglichkeiten im Bereich der Digitalisierung

- SPN Schwaben Präzision Fritz Hopf GmbH, Nördlingen

Übertragung der Ergebnisse aus dem Projekt auf andere Unternehmensbereiche und Sensibilisierung der Mitarbeiter/innen für das Thema Digitalisierung

\section{Literatur}

1. Apt E, Bovenschulte M, Priesack K et al (2018) Einsatz von digitalen Assistenzsystemen im Betrieb, iit - Institut für Innovation und Technik, Berlin

2. Riegel A, Rusch T et al (2019) SynDiQuAss - Synchronisation von Digitalisierung, Qualitätssicherung und Assistenzsystemen an Arbeitsplätzen mit geringen Automatisierungsgrad. Forschungsprojekt Transwork: Schwerpunktgruppenbuch

3. Rusch T, Kerber F (2019) Prozessmodellierung zur Integration von Assistenzsystemen an Montagearbeitsplätzen. In: GfA, Dortmund (Hrsg.): Frühjahrskongress 2019, Beitrag C.9.7 
4. Fink K, Rusch T, Merkel M et al (2020) Ein Vorgehensmodell zur Prozessevaluierung zur Integration ausgewählter kognitiver und physischer Assistenzsysteme am Montagearbeitsplatz 4.0 im Mittelstand. GfA, Dortmund (Hrsg.): Frühjahrskongress 2020, Berlin

5. Bullinger H-J (1995) Arbeitsplatzgestaltung - Personalorientierte Gestaltung marktgerechter Arbeitssysteme. Springer Vieweg, Wiesbaden

6. Sochor R, Riegel A, Merhar L et al (2019) Kognitive und physische Assistenz in der Montage. In: wt Werkstatttechnik online 109(2019) Nr. 3: 122-127

7. Kleineberg T, Hindrichsen S, Eichelberg M et al (2017) Leitfaden: Einführung von Assistenzsystemen in der Montage. Internet: https://www.th-owl.de/produktion/fachbereich/labore/ industrial-engineering/veroeffentlichungen/leitfaden-einfuehrung-von-assistenzsystemen-inder-montage. Zugriff am 06. Jani 2020

8. Rusch T, Riegel A, Kerber F et al (2019) Spezifikation und Umsetzungskonzept für standardisierte Montagearbeitsplätze mit integrierter Assistenzfunktion. In: Arbeit in der digitalisierten Welt:162-169

9. DIN EN ISO 6385 (2016) Grundsätze der Ergonomie für die Gestaltung von Arbeitssystemen. Deutsches Institut für Normung DIN EN ISO 6385:2016

10. Deuse J, Busch F, Weisner K et al (2015) Differentielle Arbeitsgestaltung durch hybride Automatisierung. Arbeit in der digitalisierten Welt, Frankfurt/New York

11. Ullrich C, Aust M, Blach R et al (2015) Assistenz- und Wissensdienste für den Shopfloor. Proceedings der Pre-Conference Workshops der 13. E-Learning Fachtagung Informatik, München

12. Bischoff J, Taphorn C, Wolter D et al (2015) Erschließen der Potenziale der Anwendung von Industrie $4.0 \mathrm{im}$ Mittelstand. agiplan, Mühlheim an der Ruhr

13. Senderek R, Geisler K (2015) Assistenzsysteme zur Lernunterstützung in der Industrie 4.0. Proceedings der Pre-Conference Workshops der 13. E-Learning Fachtagung Informatik, München

14. Ullrich A, Vladova G, Gronau N et al (2016) Akzeptanzanalyse in der Industrie 4.0-Fabrik. Ein methodischer Ansatz zur Gestaltung des organisatorischen Wandels. In: Obermaier, R. (Hrsg) Bd. Industrie 4.0 als unternehmerische Gestaltungsaufgabe, Wiesbaden: Springer Gabler, S 291-307

15. Anstadt U (1994) Determinanten der individuellen Akzeptanz bei Einführung neuer Technologien. Eine empirische arbeitswissenschaftliche Studie am Beispiel von CNC-Werkzeugmaschinen und Industrierobotern. In: Lang P (Hrsg) Bd. Arbeitswissenschaft in der betrieblichen Praxis, Frankfurt a. M.: European Verlag der Wissenschaft, S 66-94

16. Goodhue D, Thompson R (1995) Task-technology fit and individual performance. MIS Q 19(2):213-236

17. Brooke J (1994) SUS: A 'Quick and Dirty` Usability Scale. In: Jordan P, Thomas B, Weerdmeester B et al (Ed) Usability evaluation in industry. London

18. Verein Deutscher Ingenieure (1990) VDI 2860 Montage- und Handhabungstechnik; Handhabungsfunktionen, Handhabungseinrichtungen, Begriffe, Definitionen, Symbole

19. Shamsuzzoha A, Helo P (2011) Real-time Tracking and Tracing System: Potentials for the Logistics Network. Proceedings of the 2011 International Conference on Industrial Engineering and Operations Management, Kuala Lumpur 
Open Access Dieses Kapitel wird unter der Creative Commons Namensnennung 4.0 International Lizenz (http://creativecommons.org/licenses/by/4.0/deed.de) veröffentlicht, welche die Nutzung, Vervielfältigung, Bearbeitung, Verbreitung und Wiedergabe in jeglichem Medium und Format erlaubt, sofern Sie den/die ursprünglichen Autor(en) und die Quelle ordnungsgemäß nennen, einen Link zur Creative Commons Lizenz beifügen und angeben, ob Änderungen vorgenommen wurden.

Die in diesem Kapitel enthaltenen Bilder und sonstiges Drittmaterial unterliegen ebenfalls der genannten Creative Commons Lizenz, sofern sich aus der Abbildungslegende nichts anderes ergibt. Sofern das betreffende Material nicht unter der genannten Creative Commons Lizenz steht und die betreffende Handlung nicht nach gesetzlichen Vorschriften erlaubt ist, ist für die oben aufgeführten Weiterverwendungen des Materials die Einwilligung des jeweiligen Rechteinhabers einzuholen.

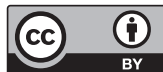

